\title{
Experimental validation of DECT based proton range prediction in inhomogeneous animal tissues using high resolution gel dosimetry
}

$\underline{\text { K. Niepel }}{ }^{1}$, M. Stanislawski ${ }^{1}$, M. Würl ${ }^{1}$, I. Yohannes ${ }^{2}$, O. Dietrich ${ }^{3}$, B. Ertl-Wagner ${ }^{3,4}$, E.Pappas $^{5}$, M. Hillbrand ${ }^{2}$, G. Landry $^{1,6}$, K.Parodi $^{1}$

${ }^{1}$ Department of Medical Physics, Ludwig-Maximilian Universität München (LMU Munich), Munich, Germany

${ }^{2}$ Rinecker Proton Therapy Center, Munich, Germany

${ }^{3}$ Department of Radiology, Ludwig-Maximilian University Hospital, Munich, Germany

\section{Motivation}

\begin{abstract}
Uncertainties in the conversion of CT number to stopping power ratio limit the dose conformity in proton therapy

-Dual energy CT (DECT) can improve the accuracy of SPR

prediction, but no high resolution verification has been done yet - Goal: Verification of various proton range conversion methods in highly inhomogeneous fresh tissue samples
\end{abstract}

${ }^{4}$ Currently at The Hospital for Sick Children, Department of Medical Imaging, University of Toronto, Toronto, Canada

Department of Biomedical Sciences, University of West Attica, Athens, Greece ${ }^{6}$ Department of Radiation Oncology, Ludwig-Maximilian University Hospital, Munich, Germany

\section{Phantom}

The phantom setup consisted of - Dosimetric polymer gel cubes [1] - Calibration boxes for each gel batch - Boxes holding animal tissue samples - Tissue equivalent materials of known composition and density (CIRS, USA)

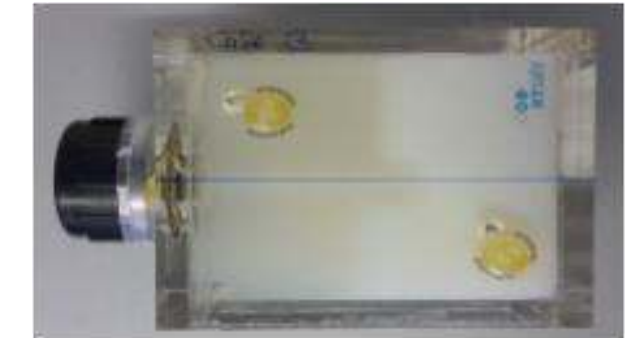

Fig 1: Irradiated gel cube with markers

\section{Materials and Methods}

\section{Measurements:}

-DECT (90kVp/150kVp with Sn filtration, Siemens Somatom Force) and SECT (120 kVp, Phillips Brilliance 16,) scans of phantoms -Water equivalent thicknesses of all samples and phantom parts, (PTW Freiburg, Germany)

-Phantom was irradiated with monoenergetic proton fields at

Rinecker Proton Therapy Center (RPTC, Munich, Germany)

-MRI of irradiated gel cubes (Siemens Magnetom Aera, 1.5 T)

\section{Simulations:}

-Monte Carlo using FLUKA [2]

-CT images imported as voxelized geometry

-HU-to-SPR conversion via look up table

-MRI scans were manually registered to FLUKA coordinates

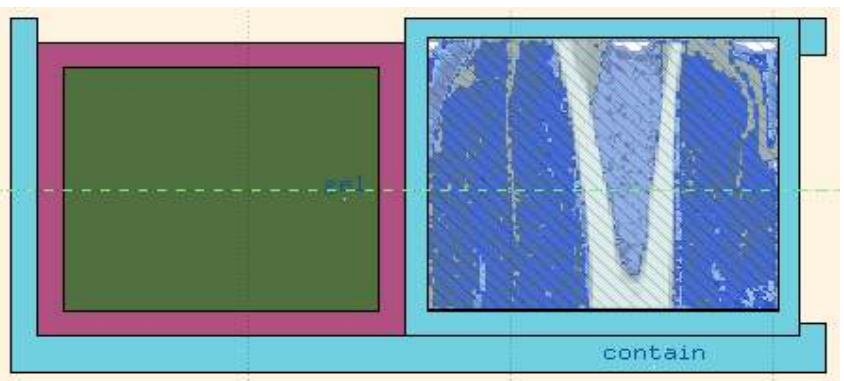

Fig 2: Combinatorial phantom geometry for inhomogeneous tissue sample (FLUKA)

\section{Results}

Homogeneous soft tissue sampl
Fig 3: Deviation of SECT-based (XiO)
and DECT-based SPR values obtained
with several methods ([3-8]) from
ground truth determined by the
peakfinder measurements. The
result based on Saito et al. is shown
for different calibrations based on
tissue equivalent materials ([8]a) and
body tissue composition ([8]b). The
central mark indicates the median,
the extension of the box covers the
interquartile range, and the extension
of the whiskers approximately covers
$99.3 \%$ of data values.

\begin{tabular}{l|l|l|}
\multicolumn{3}{c}{ Results } \\
\hline
\end{tabular}

Conclusion

Gel dosimetry showed good agreement with both peakfinder measurements and DECT-to-SPR conversion. Different conversion algorithms resulted in similarly accurate SPR prediction and standard deviations for homogeneous tissue samples.

\section{References}

$$
[1
$$

29, 2019

[2] Würl et al, PMB 61, 2016

[3] Berndt et al., PMB 62, 2017
[4] Landry et al., PMB 58, 2013 [7] Kanematsu et al., Med Phys

[5] Moehler et al., PMB 63, 2018

[6] Yang et al., PMB 55, 2010
39, 2012

[8] Saito M, Sagara S, Med Phys. 44, 2017

\section{Outlook}

Inhomogeneous tissue samples:

Fig 4: Overlaid CT scan of whole phantom and MRI scan of irradiated gel $(A)$ and corresponding Monte Carlo simulation (B) for inhomogeneous tissue sample; (beam coming from the right) More quantitative analysis is ongoing

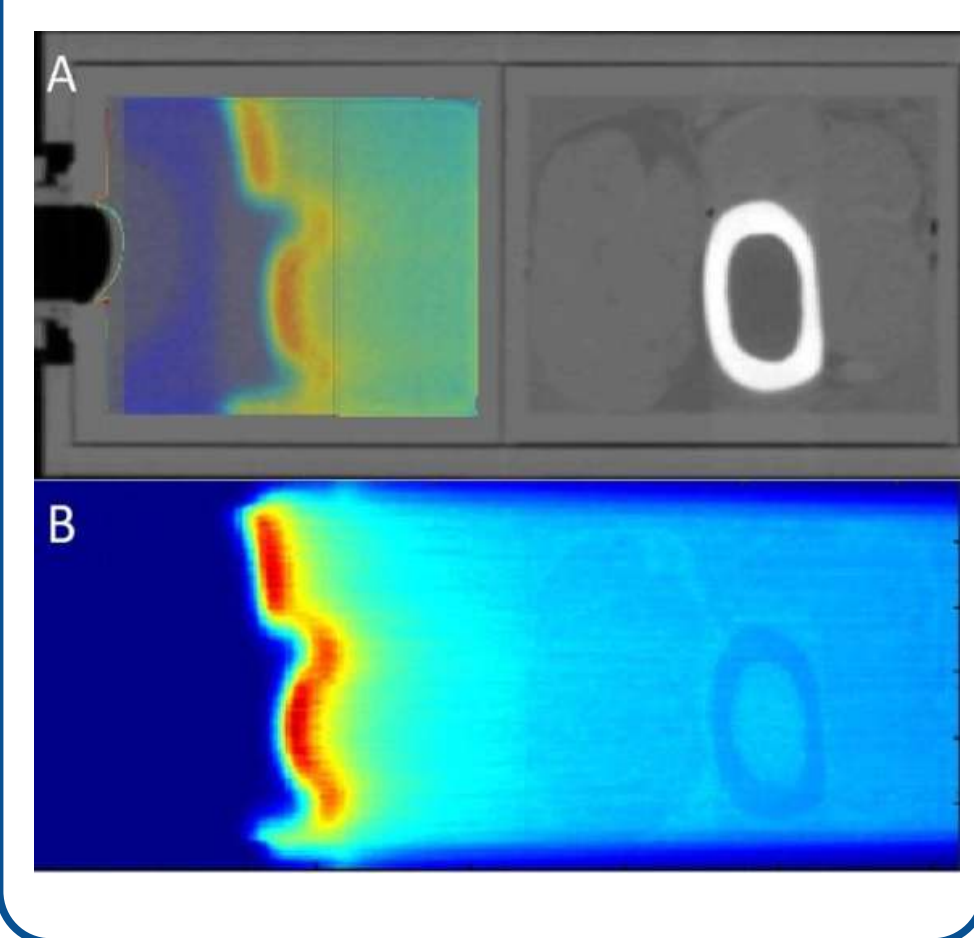

Acknowledgements

German Research Foundation (DFG) : GRK 2274, MAP Sandy Ebert; Fabian Doerringer; Christopher Kurz

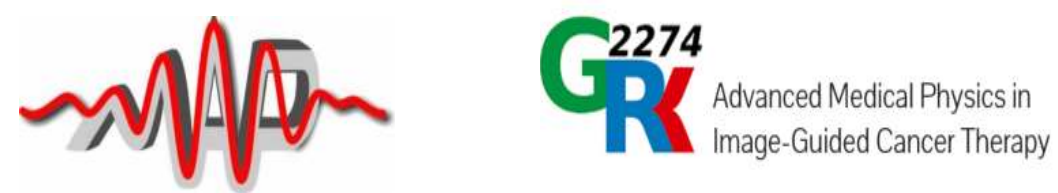

\section{Contact information:}

K.Niepel@physik.uni-muenchen.de 